\title{
Meson and baryon masses with low mode averaging
}

\author{
Gunnar Bali, Luca Castagnini*, Sara Collins \\ Institut für Theoretische Physik, Universität Regensburg, \\ 93040 Regensburg, Germany \\ E-mail: gunnar.bali@physik.uni-regensburg.de, \\ luca.castagnini@physik.uni-regensburg.de, \\ sara.collins@physik. uni-regensburg.de
}

\begin{abstract}
We describe and test a method known in the literature as low mode averaging to improve Euclidean two-point functions in lattice QCD using the low-lying eigenmodes of the Wilson-Dirac operator $D$. The contribution from the low modes is averaged over all positions of the quark sources while the contribution from high modes is calculated in the traditional way using one source point per lattice. We apply this method to different baryon and meson two-point functions and we compare the improvements using the eigenmodes of the non-hermitian operator $D$ and the eigenmodes of the hermitian operator $Q=\gamma_{5} D$. The convergence strongly depends on the parity of the states.
\end{abstract}

The XXVIII International Symposium on Lattice Field Theory, Lattice2010

June 14-19, 2010

Villasimius, Italy

\footnotetext{
*Speaker.
} 


\section{Introduction}

Solving the Dirac equation $D \psi=\eta$ may be a very time consuming task at small quark masses as the condition number of $D$ is proportional to the inverse of the quark mass. Some approaches involve the computation of the low-lying eigenvectors of $\gamma_{5} D$ and use these to deflate the Dirac operator, see e.g. ref. [1, 2]. These eigenvectors can also be used to reduce the noise of the signal with a technique known in the literature $[3,4]$ as low mode averaging (LMA). This consists of decomposing the quark propagator into a sum of high and low modes $\psi=\psi_{\text {high }}+\psi_{\text {low }}$ and in averaging the low mode contribution over all lattice points. We define the LMA two-point function as,

$$
C_{\mathrm{LMA}}(t)=C_{\mathrm{low}}(t)+C^{\mathrm{pa}}(t)-C_{\mathrm{low}}^{\mathrm{pa}}(t),
$$

where $C^{\mathrm{pa}}$ is the exact point-to-all correlation function, calculated for a single source point. This definition satisfies $\left\langle C_{\mathrm{LMA}}(t)\right\rangle=\left\langle C^{\mathrm{pa}}(t)\right\rangle$, however, the errorbars are reduced, due to more sampling per lattice. In the present work we apply the LMA technique to meson and baryon two-point functions with degenerate quark masses. We study the improvement as a test for future work on hadron spectroscopy and three-point functions.

\section{Mesons}

The present study is based on 100 configurations with lattice volume $V=16^{3} \times 32$ generated with the quenched Wilson action at $\beta=6.0175$ using Chroma [5]. This corresponds to a lattice spacing of $a \approx 0.2093 \sigma^{-1 / 2} \approx 0.093 \mathrm{fm}$. For each configuration we computed the lowest 30 eigenvectors of the massive hermitian Dirac operator $\gamma_{5} D\left|u_{i}\right\rangle=\lambda_{i}\left|u_{i}\right\rangle$ using the algorithm by Kalkreuter and Simma [6] at $\kappa=0.1557$ corresponding to $m_{\pi} \approx 425 \mathrm{MeV}$ and $m_{\pi} L \approx 3.2$. We can reconstruct the contribution of the low modes to the quark propagator as [7], $D_{\text {low }}^{-1}=\sum_{i} \frac{1}{\lambda_{i}}\left|u_{i}\right\rangle\left\langle u_{i}\right| \gamma_{5}$ while $C_{\text {low }}(t)$ is given by,

$$
C_{\mathrm{low}}(t)=\sum_{i, j, x, \mathbf{y}} \frac{1}{\lambda_{i} \lambda_{j}}\left\langle u_{j}(x)\left|\gamma_{5} \Gamma\right| u_{i}(x)\right\rangle\left\langle u_{i}(y)\left|\gamma_{5} \Gamma\right| u_{j}(y)\right\rangle
$$

where $y_{4}=x_{4}+t$.

Clearly the LMA technique works best when the low modes are dominant. This happens for the $\pi$ as can be seen from fig. 1 (left) where we compare the smeared-smeared $\pi$ point-to-all correlation function with the low mode contribution. However, for positive parity mesons (see fig. 1 (right) for the $a_{0}$ ), the low modes saturate the two-point function very slowly. This can also be seen by comparing the LMA and point-to-all smeared-smeared effective masses of fig. 2 for negative parity mesons with the positive parity ones of fig. 3 . In the case of the $a_{0}$ the low mode contribution even has a wrong curvature in the central region, see fig. 1 (right).

The meson correlators differ in the $\Gamma$ structure of the interpolating fields: for the $\pi$ (the best case) we have a total $\Gamma$ product $\gamma_{5} \Gamma=\mathbb{1}$ in eq. (2.1) while for the $a_{0}$ this reads $\gamma_{5} \Gamma=\gamma_{5}$. One way to get rid of a $\gamma_{5}$ factor is to calculate the right and left eigenvectors of the non-hermitian Dirac operator, $D\left|r_{i}\right\rangle=\lambda_{i}\left|r_{i}\right\rangle,\left\langle\ell_{i}\right| D=\lambda_{i}\left\langle\ell_{i}\right|$ and to construct the quark propagator as [8,9], $D_{\text {low }}^{-1}=$ $\sum_{i} \frac{1}{\lambda_{i}}\left|r_{i}\right\rangle\left\langle\ell_{i}\right|$. In this way the meson two-point function becomes, 

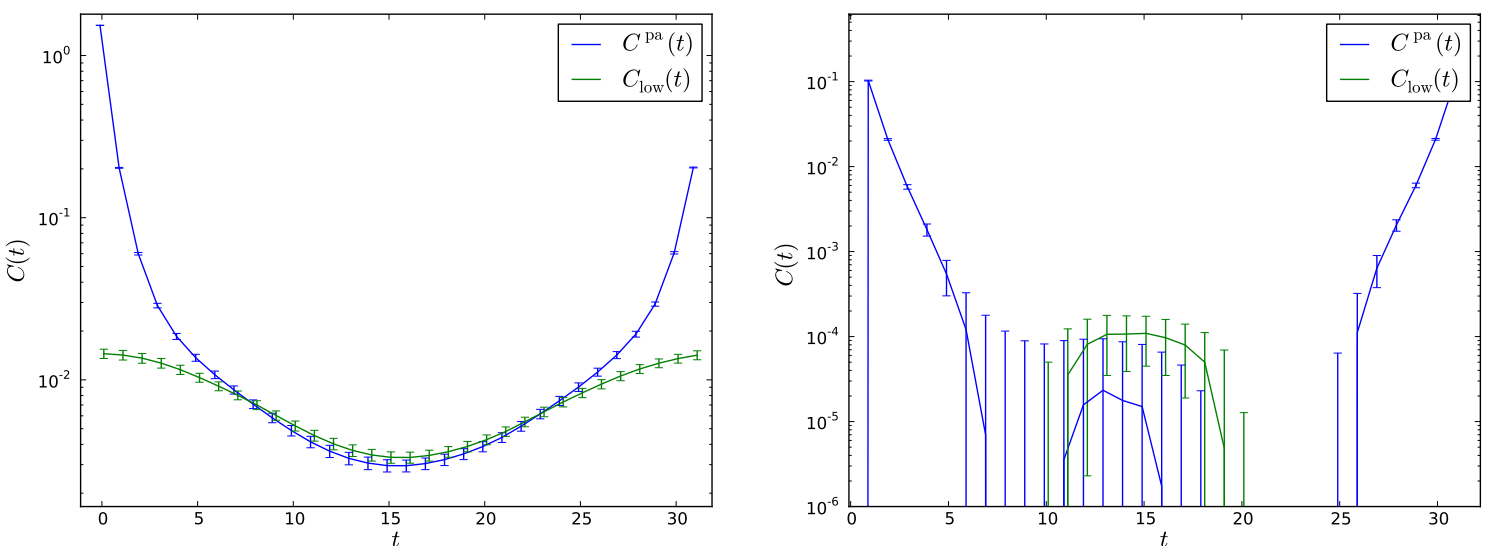

Figure 1: Low mode saturation (30 modes): $\pi\left(J^{P C}=0^{-+}\right.$, left $)$and $a_{0}\left(J^{P C}=0^{++}\right.$, right $)$two-point functions.
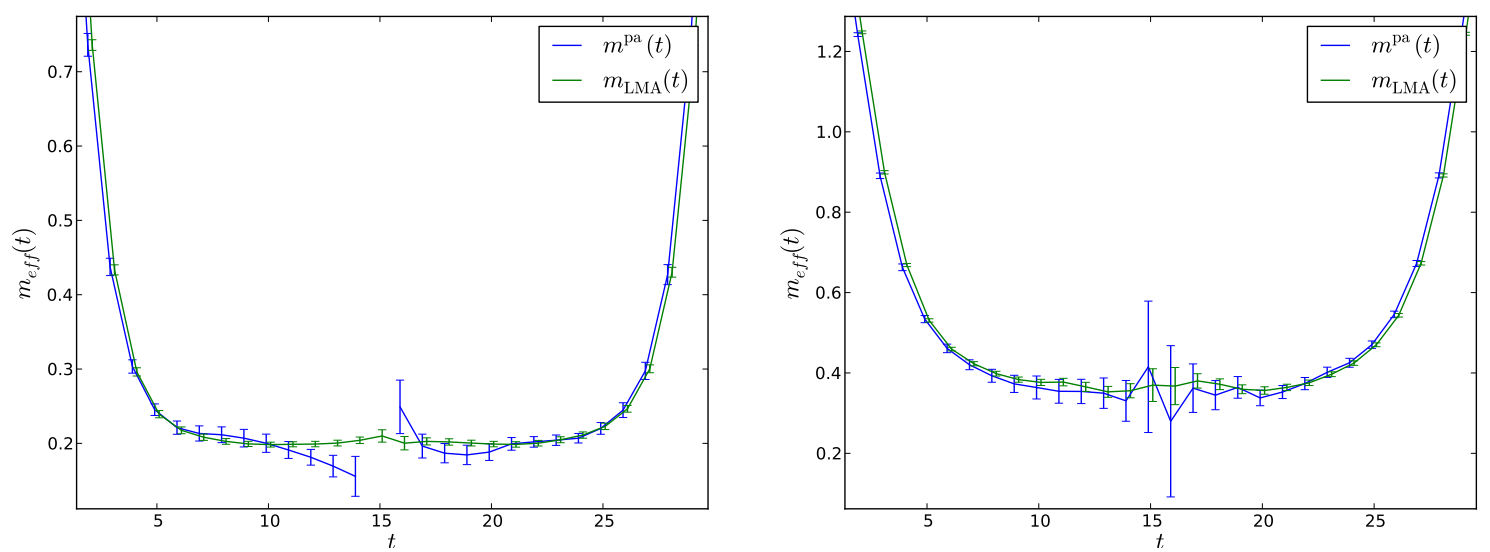

Figure 2: Effective masses: $\pi\left(J^{P C}=0^{-+}\right.$, left $)$and $\rho\left(J^{P C}=1^{--}\right.$, right $)$
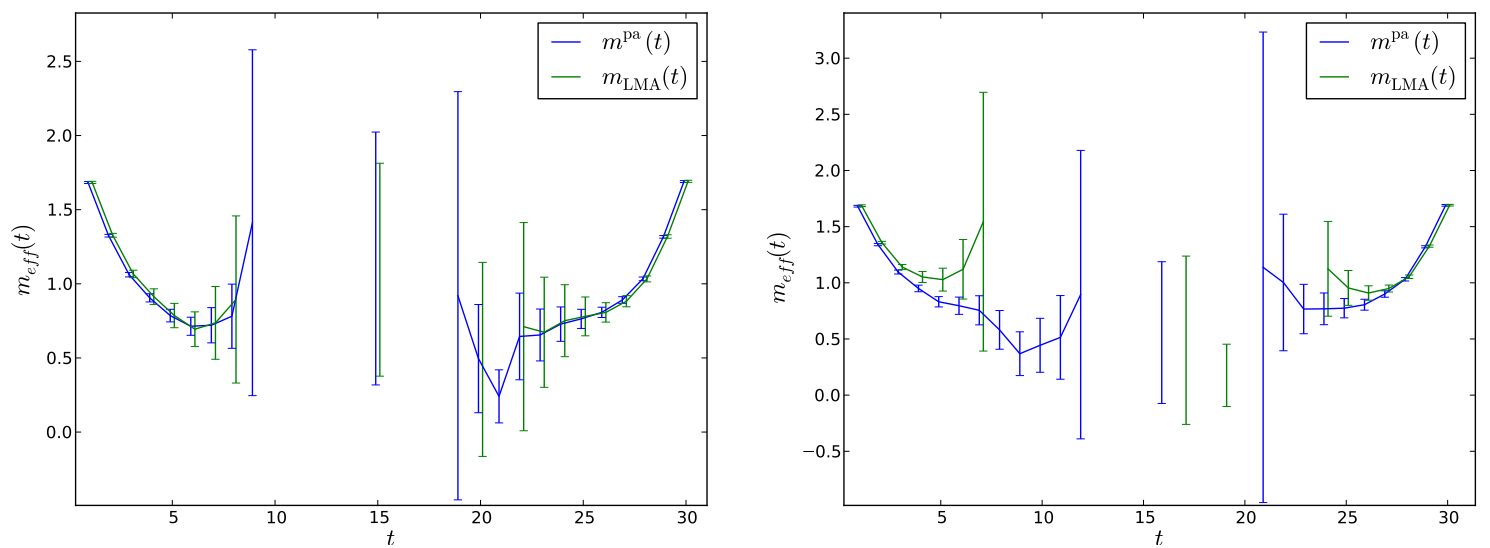

Figure 3: Effective masses: $a_{1}\left(J^{P C}=1^{++}\right.$, left $)$and $b_{1}\left(J^{P C}=1^{+-}\right.$, right $)$.

$$
C_{\mathrm{low}}(t)=\sum_{i, j, x, \mathbf{y}} \frac{1}{\lambda_{i} \lambda_{j}}\left\langle\ell_{j}(x)|\Gamma| r_{i}(x)\right\rangle\left\langle\ell_{i}(y)|\Gamma| r_{j}(y)\right\rangle
$$

Note that $\left\langle\ell_{i} \mid r_{j}\right\rangle=\delta_{i j}$ and it can easily be seen that due to the property $D^{\dagger}=\gamma_{5} D \gamma_{5}, \lambda_{i}^{*}$ is an 
eigenvalue whenever $\lambda_{i}$ is an eigenvalue, with a left eigenvector $\left\langle\tilde{\ell}_{i}\right|=\left\langle r_{i}\right| \gamma_{5}$ and a right eigenvector $\left|\tilde{r}_{i}\right\rangle=\gamma_{5}\left|\ell_{i}\right\rangle$.

\begin{tabular}{|c|c|c|}
\hline & $m$ & $m_{\text {LMA }}$ \\
\hline$\pi$ & $0.2003(49)$ & $0.2019(36)$ \\
\hline$\rho$ & $0.3965(66)$ & $0.3952(41)$ \\
\hline
\end{tabular}

Table 1: Fitted meson masses in lattice units.

We used the Arnoldi method implemented in the ARPACK library to compute the non-hermitian eigenvectors. This method is much slower than that of Kalkreuter and Simma. However, the eigenvectors are independent of the hopping parameter $\kappa$ and when changing the quark mass we only need to rescale the eigenvalues $\lambda$. Unfortunately, we could not find any significant improvement using 15 pairs of non-hermitian low modes over the standard point-to-all method. This may be related to the fact that the ratios $\left|\lambda_{30}\right| /\left|\lambda_{1}\right|$ were found to be by factors of approximately five smaller in the non-hermitian case than for the hermitian case.

From the fit to the correlators we found a $30 \%$ improvement on the extrapolated $\pi$ and $\rho$ meson masses for the hermitian LMA, as displayed in table 1.

We also applied the method to the local axialvector current (fig. 4), to test the improvement on the pion decay constant $f_{\pi}$. Following the conventions of ref. [10] we set,

$$
m_{\pi} f_{\pi}=\left\langle 0\left|\gamma_{4} \gamma_{5}\right| \pi\right\rangle=-2 \kappa \frac{\sqrt{2 m_{\pi}} A_{A P}}{\sqrt{A_{P P}}} .
$$

We denote the amplitudes of correlation functions with a smeared pseudoscalar sink and a local axial-vector source as $A_{A P}$ and with a local pseudoscalar source as $A_{P P}$. The improvement of LMA is very striking in this case,

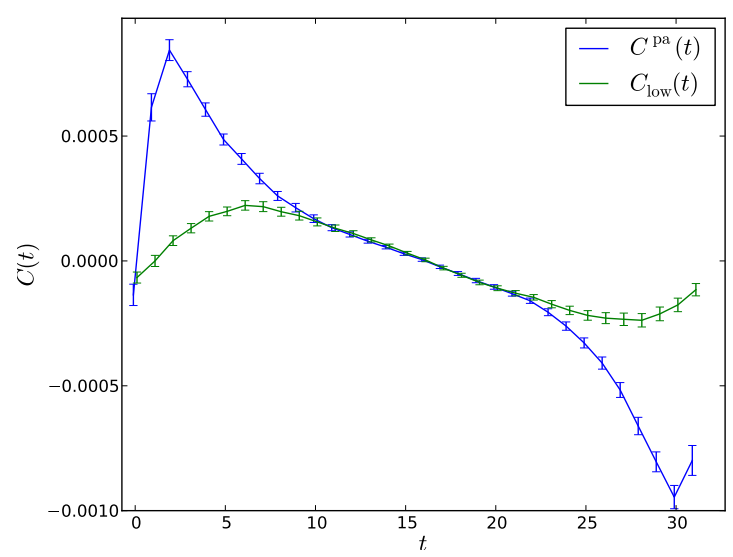

Figure 4: Local axial-vector current.

$$
f_{\pi}=0.079(27), \quad f_{\pi, \mathrm{LMA}}=0.082(12)
$$

\section{Baryons}

The low mode averaging technique for baryons is more challenging due to the way the interpolators are contracted. For the nucleon $N$, the $\Delta^{+}$and the $\Lambda$ we use the following interpolators,

$$
\begin{aligned}
N(x) & =\varepsilon_{a b c} u(x)_{a}\left(u(x)_{b}^{T} C \gamma_{5} d(x)_{c}\right) \\
\Delta^{+}(x) & =\varepsilon_{a b c}\left[2\left(u(x)_{a}^{T} C \gamma_{\mu} d(x)_{b}\right) u(x)_{c}+\left(u(x)_{a}^{T} C \gamma_{\mu} u(x)_{b}\right) d(x)_{c}\right] \\
\Lambda(x) & =\varepsilon_{a b c}\left[2\left(u(x)_{a}^{T} C \gamma_{5} d(x)_{b}\right) s(x)_{c}+\left(u(x)_{a}^{T} C \gamma_{5} s(x)_{b}\right) d(x)_{c}-\left(d(x)_{a}^{T} C \gamma_{5} s(x)_{b}\right) u(x)_{c}\right],
\end{aligned}
$$

where $C$ is the charge conjugation operator. Note that we only study the mass-degenerate case, $m_{u}=m_{d}=m_{s}$. In the following, we consider the contractions for the example of the nucleon two-point function,

$$
\langle N(y) \bar{N}(x)\rangle=\left\langle\varepsilon_{a b c} \varepsilon_{a^{\prime} b^{\prime} c^{\prime}}\left(C \gamma_{5}\right)_{\alpha \beta}\left(C \gamma_{5}\right)_{\alpha^{\prime} \beta^{\prime}}\left(P_{ \pm}\right)_{\gamma \gamma^{\prime}} d(y)_{b^{\prime} \beta^{\prime}} \bar{d}(x)_{b \beta} u(y)_{a^{\prime} \alpha^{\prime}} \bar{u}(x)_{a \alpha} u(y)_{c^{\prime} \gamma^{\prime}} \bar{u}(x)_{c \gamma}\right\rangle,
$$


where $P_{ \pm}=\frac{1}{2}\left(\mathbb{1} \pm \gamma_{4}\right)$ is the parity projector.

The expression eq. (3.4) can be written in terms of the left and right eigenvector components in a way that the source term is decoupled from the sink term:

$$
\begin{aligned}
\langle N(y) \bar{N}(x)\rangle= & \sum_{i, j, k} \frac{1}{\lambda_{i} \lambda_{j} \lambda_{k}}\left\{\varepsilon_{a^{\prime} b^{\prime} c^{\prime}}\left(C \gamma_{5}\right)_{\alpha^{\prime} \beta^{\prime}}\left[r_{i, b^{\prime}, \beta^{\prime}}(y) r_{j, a^{\prime}, \alpha^{\prime}}(y) r_{k, c^{\prime}, \gamma^{\prime}}(y)\right]\right\}\left(P_{ \pm}\right) \gamma \gamma^{\prime} \\
& \times\left\{\varepsilon_{a b c}\left(C \gamma_{5}\right)_{\alpha \beta} \ell_{i, b, \beta}^{*}(x)\left[\ell_{j, a, \alpha}^{*}(x) \ell_{k, c, \gamma}^{*}(x)-\ell_{j, c, \gamma}^{*}(x) \ell_{k, a, \alpha}^{*}(x)\right]\right\}
\end{aligned}
$$

For the hermitian case, $\gamma_{5} D\left|u_{i}\right\rangle=\lambda_{i}\left|u_{i}\right\rangle,\left|r_{i}\right\rangle=\left|u_{i}\right\rangle$ and $\left\langle\ell_{i}\right|=\left\langle u_{i}\right| \gamma_{5}$. The cost of computing the above expression increases with the third power of the number of eigenmodes used. To optimize this computation, we split up each eigenvector $r$ into twelve $r_{a, \alpha}$ spin-colour components and store them as lattice objects. Multiplications like $r_{i, a, \alpha} r_{j, b, \beta}$ are optimized in QDP++. With these and other optimizations the cost of the contractions for 30 eigenmodes becomes negligible relative to the cost of solving for the propagator.
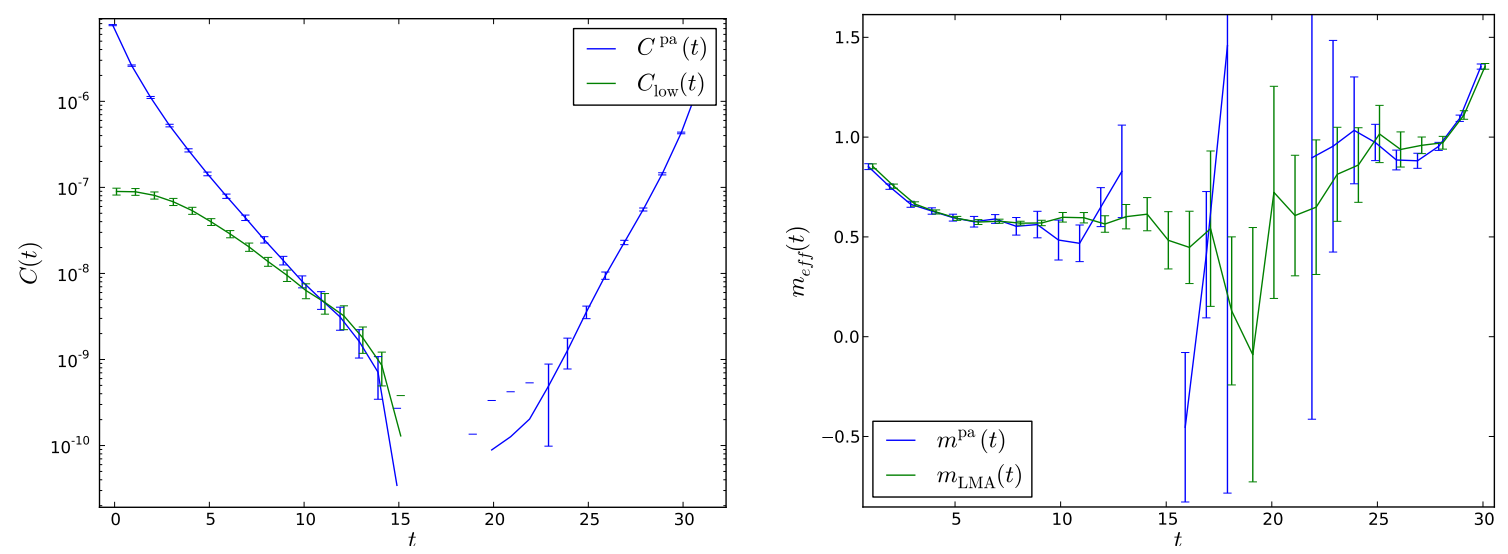

Figure 5: Nucleon $\left(J^{P}=\frac{1}{2}^{+}\right)$two-point function and effective masses. The backwards propagating state is the $N^{*}\left(J^{P}=\frac{1}{2}^{-}\right)$.
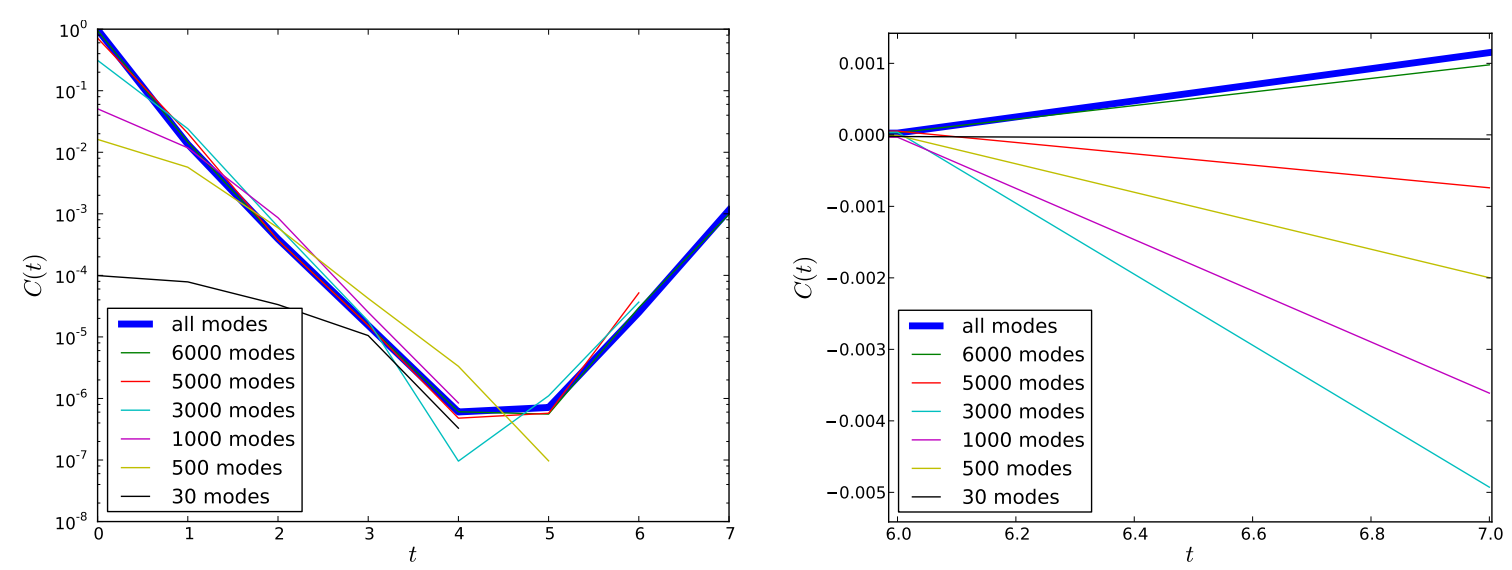

Figure 6: Low mode contribution to the nucleon two-point function on a $4^{3} \times 8$ lattice. On the right hand side the $t=6,7$ data are shown without the logarithmic scale.

In fig. 5 we show the low mode saturation of the nucleon two-point function and the LMA improvement of the corresponding effective mass. The low mode contribution for the $N^{*}$ turns 
out to be negative and is not visible on the logarithmic scale. To gain a better understanding of this behaviour we investigated a small $4^{3} \times 8$ lattice volume where we calculated all the 6144 eigenvectors of the Wilson operator using the LAPACK library. In fig. 6 we display the nucleon two-point function $C_{\text {low }}(t)$ on such a single configuration for different numbers of eigenmodes. For the positive parity state that propagates from $t=0$ into the forward direction the low modes are dominant and quickly saturate the correct correlation function. Conversely, for the backward propagating states even the sign is wrong until over $85 \%$ of the modes are summed up. Eventually, after all modes are included, the correct result is obtained. We also tested non-hermitian LMA for baryons. This did not solve the sign problem for negative parity states and, as in the mesonic case, no improvement over the conventional point-to-all method was found.
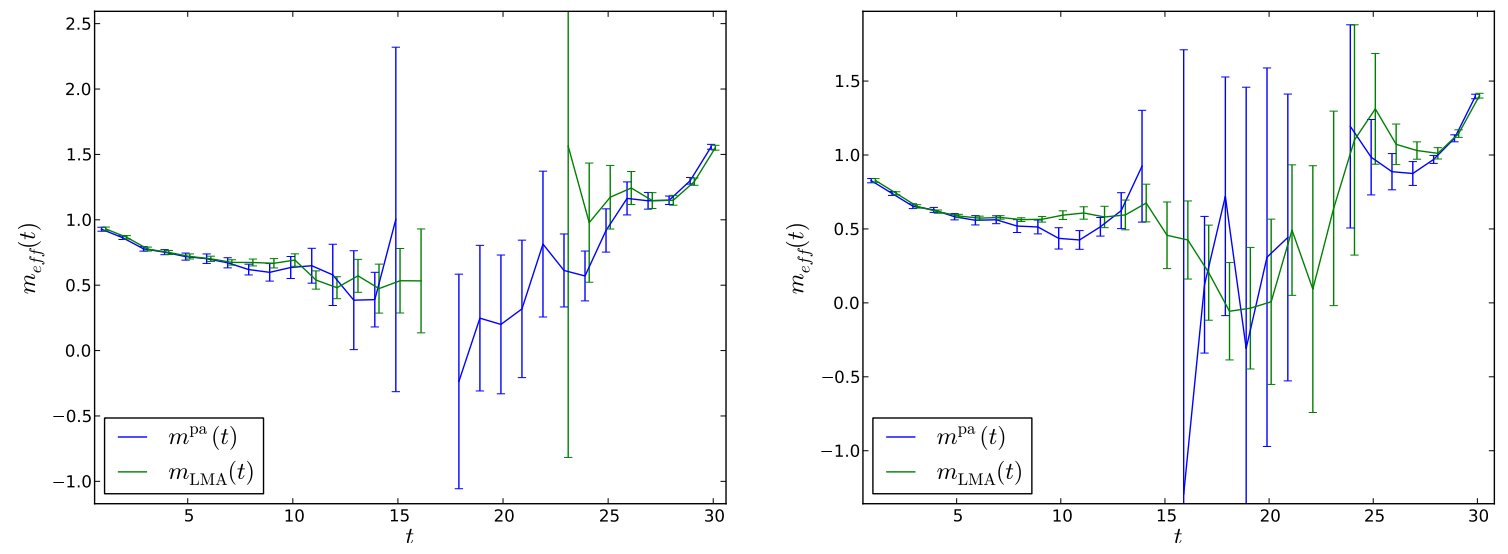

Figure 7: Effective masses for the $\Delta^{+}\left(J^{P}=\frac{3}{2}^{+}\right.$, left $)$and $\Lambda\left(J^{P}=\frac{1}{2}^{-}\right.$, right $)$.

For the $\Delta^{+}$and the $\Lambda$ a behaviour similar to that of the nucleon was found. In fig. 7 we display the effective mass plots. The negative parity partners are not well approximated by the lowest modes that exhibit the wrong sign. However, LMA reduces the statistical errors for the forward propagating states. The errors on the fitted positive parity baryon masses are reduced by factors of roughly two, using the hermitian LMA method, see table 2 .

\begin{tabular}{|c|c|c|}
\hline & $m$ & $m_{\text {LMA }}$ \\
\hline$p$ & $0.604(16)$ & $0.595(7)$ \\
\hline$\Delta^{+}$ & $0.717(20)$ & $0.713(12)$ \\
\hline$\Lambda$ & $0.586(17)$ & $0.592(9)$ \\
\hline
\end{tabular}

Table 2: Fitted baryon masses in lattice units for $m_{u}=m_{d}=m_{s}$.

\section{Conclusions}

We confirm that the hermitian low mode averaging technique is an efficient method to reduce the noise of two-point functions and of the fitted hadron masses. It works well for negative parity mesons and very well for positive parity baryons but it fails completely for the opposite parity cases where low mode saturation does not set in at all. Non-hermitian low mode averaging was inefficient for all particles studied, due to its slower saturation behaviour.

It should be pointed out that computing the low lying eigenmodes is computationally demanding. Preliminary tests on a $24^{3} \times 48$ volume with $m_{\pi} \approx 420 \mathrm{MeV}\left(m_{\pi} L \approx 4.8\right)$ show that it is more efficient to increase the number of point sources than to compute the eigenvectors, to reduce 
the errors on meson masses. For baryons the LMA is cost-efficient. If one further decreases the quark mass then on one hand the costs of computing propagators will drastically increase while on the other hand low mode saturation may set in faster, making this method even more efficient. Moreover, eigenvectors may be recycled for deflating the Dirac operator, in particular if multiple source points per configuration are used, see e.g. refs. [1,2]. In this case the overhead of LMA is negligible.

\section{Acknowledgments}

We thank Jacques Bloch for discussions. The simulations were run on Regensburg's Athene HPC cluster. Sara Collins was supported by the Claussen-Simon-Foundation (Stifterverband für die deutsche Wissenschaft). We acknowledge support from Deutsche Forschungsgemeinschaft (Sonderforschungsbereich/Transregio 55) and the European Union grant 238353 (ITN STRONGnet).

\section{References}

[1] R. B. Morgan and W. Wilcox, Deflated iterative methods for linear equations with multiple right-hand sides, arXiv:math-ph/0405053.

[2] A. Stathopoulos and K. Orginos, Computing and deflating eigenvalues while solving multiple right hand side linear systems in Quantum Chromodynamics, arXiv:0707.0131 [hep-lat] .

[3] T. A. DeGrand and S. Schäfer, Improving meson two-point functions in lattice QCD, Comput. Phys. Commun. 159 (2004) 185 [arXiv: hep-lat/ 0401011$].$

[4] L. Giusti, P. Hernandez, M. Laine, P. Weisz and H. Wittig, Low-energy couplings of QCD from current correlators near the chiral limit, JHEP 0404 (2004) 013 [arXiv: hep-lat / 0402002 ].

[5] R. G. Edwards and B. Joo [SciDAC, LHP and UKQCD Collaborations], The Chroma software system for lattice QCD, Nucl. Phys. Proc. Suppl. 140 (2005) 832 [arXiv : hep-lat / 0409003 ]; C. McClendon, Optimized lattice QCD kernels for a Pentium 4 cluster, Jlab preprint, JLAB-THY-01-29, http: //www.jlab.org/ edwards/qcdapi/reports/dslash_p4.pdf

[6] T. Kalkreuter and H. Simma, An accelerated conjugate gradient algorithm to compute low lying eigenvalues: a study for the Dirac operator in SU(2) lattice QCD”, Comput. Phys. Commun. 93 (1996) 33 [arXiv: hep-lat/9507023].

[7] H. Neff, N. Eicker, T. Lippert, J. W. Negele and K. Schilling, On the low fermionic eigenmode dominance in QCD on the lattice, Phys. Rev. D 64 (2001) 114509 [arXiv: hep-lat/ 0106016 ].

[8] G. S. Bali, S. Collins and A. Schäfer, Effective noise reduction techniques for disconnected loops in Lattice QCD, Comput. Phys. Commun. 181 (2010) 1570 [arXiv:0910.3970 [hep-lat]].

[9] V. Guerrero, R. B. Morgan and W. Wilcox, Eigenspectrum noise subtraction methods in lattice QCD, PoS LAT2009 (2009) 041 [arXiv: 1001.4366 [hep-lat ] ].

[10] M. Göckeler, R. Horsley, H. Perlt, P. E. L. Rakow, G. Schierholz, A. Schiller and P. Stephenson, Scaling of non-perturbatively $O(a)$ improved Wilson fermions: hadron spectrum, quark masses and decay constants, Phys. Rev. D 57 (1998) 5562 [arXiv: hep-lat/9707021]. 